\title{
Нейрофизиологические механизмы аддиктивного поведения и употребления психоактивных веществ в подростковом возрасте: возможности профилактики и терапии
}

Анцыборов А.В. ${ }^{1,3}$, Камплицкая О.В. ${ }^{2}$, Овсянников М.В. ${ }^{3}$, Стадник К.В. ${ }^{3}$, Панченко Л.Ф. ${ }^{4}$

\author{
' Медицинский центр «Альфа Центр Здоровья», ООО «Медицина Альфа Страхования». \\ 344022, Ростов-на-Дону, ул. Социалистическая, д. 154А \\ ${ }^{2}$ Государственное бюджетное учреждение Ростовской области «Психоневрологический диспансер». \\ 344010, Ростов-на-Дону, ул. Семашко, д. 120 \\ ${ }^{3}$ Федеральное государственное бюджетное образовательное учреждение высшего образования «Ростовский \\ государственный медицинский университет» Министерства здравоохранения Российской Федерации. \\ 344022, Ростов-на-Дону, Нахичеванский пер., д. 29 \\ ${ }^{4}$ Федеральное государственное бюджетное научное учреждение \\ «Научно-исследовательский институт общей патологии и патофизиологии». \\ 125315, Москва, ул. Балтийская, д. 8
}

Поведенческие аддикции подростков, включающие употребление психоактивных веществ, являются одними из основных проблем здравоохранения большинства стран мира, в том числе России. Подростковый возраст - это период динамичных физиологических, психологических, и поведенческих изменений. Данный период также связан с повышенным риском употребления психоактивных веществ, и формированием различных аддиктивных расстройств. В подростковом возрасте происходящие изменения в нейронных системах вознаграждения, мотивации, когнитивного контроля и сопротивления стрессовым воздействиям способствуют формированию повышенного риска употреблении различных психоактивных веществ, и нехимических вариантов аддиктивной патологии. Современные патофизиологические модели возникновения аддиктивной патологии в подростковом возрасте включают данные о аллостатических изменениях функций и структуры дофаминергической системы среднего мозга, нейропластичности, связанной со стрессом, и нарушениии баланса между когнитивным контролем и прочессами в системе вознаграждения. Результаты генетических и эпигенетических исследований, изучение промежуточных фенотипов / эндофенотипов могут помочь в изучении детей и подростков, входящих в группу риска возникновения аддиктивных расстройств.

Ключевые слова: подростковый возраст; нейрофизиология; аддиктивное поведение; употребление психоактивных веществ; онтогенез.

Для цитирования: Анцыборов А.В., Камплицкая О.В., Овсянников М.В., Стадник К.В., Панченко Л.Ф. Нейрофизиологические механизмы аддиктивного поведения и употребления психоактивных веществ в подростковом возрасте: возможности профилактики и терапии. Патогенез. 2019; 17(4): 21-32

DOI: $10.25557 / 2310-0435.2019 .04 .21-32$

Для корреспонденции: Камплицкая Оксана Владимировна, e-mail: kamplicka@mail.ru Финансирование. Исследование не имеет спонсорской поддержки. Конфликт интересов. Авторы заявляют об отсутствии конфликта интересов.

Поступила: 22.05.2019

\section{Neurophysiological mechanisms of addictive behavior and substance use in adolescence: prevention and therapy}

Antsiborov A.V. ${ }^{1,3}$, Kamplitskaya O.V. ${ }^{2}$, Ovsyannikov M.V. ${ }^{3}$, Stadnik K.V. ${ }^{3}$, Panchenko L.F. ${ }^{4}$

\footnotetext{
${ }^{1}$ The Alpha Health Center Medical Center, Medicine Alpha Insurance Medicine, LLC, Sotsialisticheskaya Str. 154A, Rostov-on-Don 344022, Russian Federation

${ }^{2}$ Rostov Regional Psychoneurological Dispensary,

Semashko Str. 120, Rostov-on-Don 344010, Russian Federation

${ }^{3}$ Rostov State Medical University,

Nahichevanskij Pereulok 29, Rostov-on-Don 344022, Russian Federation

${ }^{4}$ Institute of General Pathology and Pathophysiology,

Baltijskaya Str. 8, Moscow 125315, Russian Federation
}

The use of psychoactive substances as a part of behavioral addictions of adolescents is a medical issue in most of countries all over the world, including Russia. Adolescence is a period of dynamic physiological, psychological, and behavioral changes. This period is also associated with increased risk of substance abuse and formation of various addictive disorders. In adolescence, changes in neural systems of reward, motivation, cognitive control, and resistance to stress contribute to increased risk of using various psychoactive substances and of non-chemical variants of addictive pathology. Current pathophysiological models of addictive pathology in adolescence include data on allostatic changes in functions and structure of the mid-brain dopaminergic 
system, neuroplasticity related with stress, and imbalance between cognitive control and processes in the reward system. Results of genetic and epigenetic studies and investigation of intermediate phenotypes / endophenotypes can help to study children and adolescents at risk of addictive disorders.

Key words: adolescence; neurophysiology; addictive behavior; using of psychoactive substances; ontogenesis.

For citation: Antsiborov A.V., Kamplitskaya O.V., Ovsyannikov M.V., Stadnik K.V., Panchenko L.F. [Neurophysiological mechanisms of addictive behavior and substance use in adolescence: prevention and therapy]. Patogenez [Pathogenesis]. 2019; 17(2): 21-32 (in Russian)

DOI: $10.25557 / 2310-0435.2019 .04 .21-32$

For correspondence: Kamplitskaya Oksana Vladimirovna, e-mail: kamplicka@mail.ru

Funding. The study had no sponsorship.

Conflict of interest. The authors declare no conflict of interest. Received: 22.05.2019

\section{Введение}

Подростковый возраст изобилует различными биологическими и психологическими изменениями, включающими следующие факторы: 1) физическое и половое созревание; 2) формирование идентичности подростка и его индивидуализация; 3) повышение самостоятельности и ответственности; 4) повышение роли социальных взаимоотношений со сверстниками, формирование романтических интересов; 5) повышение роли исследовательского поведения [1]. Несмотря на то, что подростковый возраст является одним из «самых здоровых» периодов в отношении острых и хронических соматических заболеваний, он тем не менее отмечен 2-3-кратным увеличением смертности, в сравнении с детским и взрослым возрастом [2]. Основными причинами смерти в подростковом возрасте, являются ДТП, самоубийства и убийства. Все перечисленные причины связаны с функцией когнитивного контроля и импульсивным/рискованным поведением, которые могут нарушаться/усиливаться в связи с употреблением психоактивных веществ.

Недавно проведенные исследования показывают, что более $80 \%$ подростков пробуют наркотики или алкоголь до периода совершеннолетия [3]. Начало употребления различных психоактивных веществ (ПАВ), быстрый переход к более рискованным формам употребления, а также пик формирования аддиктивной патологии, приходятся именно на подростковый возраст. В подростковой среде отмечаются более высокие показатели проблемного употребления ПАВ и частоты возникновения аддиктивных расстройств в сравнении с детьми и пожилыми людьми [4]. Раннее начало употребления ПАВ может выступать предиктором развития наркотической зависимости, различной степени тяжести психических расстройств, приводить к социальной и трудовой дезадаптации. Наряду с употреблением ПАВ риск формирования других форм аддиктивной патологии (нехимические формы зависимости) также повышен в подростковом возрасте.

Понимание нейрофизиологической основы аддиктивных расстройств облегчит выявление подростков, находящихся в группе риска возникновения данной патологии, и связанных с ней последствий для соматического и психического здоровья, а также поможет в разработке эффективных стратегий терапевтических вмешательств и профилактических мер. В настоящем обзоре рассматриваются нейрофизиологические корреляции употребления ПАВ и других аддиктивных расстройств в подростковом возрасте, а также различные биологические модели аддиктивной патологии. Отдельное внимание уделено биологическим факторам риска возникновения и прогрессирования наркотической зависимости в подростковом возрасте, нейротоксическим эффектам ПАВ, перспективам терапевтического вмешательства, вопросам профилактики.

Аддиктивные расстройства: химические и поведенческие зависимости

Термин аддикция (зависимость) происходит от латинского слова addicere, означающего «порабощенный (рабский)», или «ограниченный (связанный)». Ключевыми особенностями любой аддикции является компульсивное поведение (например, употребление наркотических веществ), явления крейвинга (англ. craving - страстное желание, тяга), утрата ситуационного поведенческого контроля, постоянное употребление ПАВ, несмотря на неблагоприятные последствия [5]. До настоящего времени продолжаются дискуссии по поводу расширения понятия аддикции. Предлагается включить в данное понятие нехимические формы аддикции (аддиктивного поведения), которые носят ярко выраженный компульсивный характер. К основным аддиктивным формам поведения принято относить: азартные игры (гемблинг), еду (пищевая зависимость), секс (сексуальное аддиктивное поведение), покупки (компульсивный шопинг), интернет (интернет-зависимость) и видеоигры (гейминг). Все перечисленные формы имеют общие клинические проявления и стереотип формирования, сходный с таковым при употреблении ПАВ.

Большое количество людей играет в азартные игры, пользуются интернетом, играют в видеоигры, делают различные покупки в реальном и виртуальном пространстве. При этом их поведение носит полностью адаптивный характер. Для тех, у кого нарушен импульсный контроль, описанные выше привычные действия, могут представлять собой предикторы поведенческих (нехимических) аддикций, с соответствующи- 
ми неблагоприятными последствиями. Поведенческие аддикции в подростковом возрасте могут развиваться параллельно с расстройствами, связанными с употреблением ПАВ, или выступать в роли мощных предиспонирующих факторов употребления ПАВ - в сравнении со взрослыми.

Распространенность гемблинга среди подростков, в 2-4 раза выше, чем у взрослых. Придание геймерству статуса киберспорта, и массовое распространение широкополосного доступа в интернет привело к еще большему распространению аддиктивных расстройств среди подростков, ухудшая основные показатели здоровья и социального функционирования [6-8]. За последние несколько десятилетий резко возросли показатели ожирения среди детей и подростков, что отчасти объясняется чрезмерным потреблением продуктов фаст-фуда (доступных для онлайн заказов) и малоподвижным образом жизни [9].

Стереотип и динамика развития химических и нехимических форм аддикции являются важными компонентами оценки при структурно-динамическом анализе, особенно в подростковом возрасте. Именно в данный период аддиктивная патология может носить синдромально незавершенный характер, не достигая «большого наркоманического синдрома», и не соответствовать всем критериям зависимости. При этом имеющиеся расстройства могут приводить к ухудшению состояния здоровья, и социального функционирования [10].

\section{Патофизиологические механизмы аддиктивных расстройств}

Большинство существующих в настоящее время патофизиологических моделей, в целом, позволяют объяснить механизмы употребления ПАВ, и формирование аддиктивной патологии, а также возможные риски возникновения аддиктивных расстройств [5]. Данные модели не являются взаимоисключающими, а скорее дополняют друг друга. Большая часть из них исследует различные аспекты аддиктивного поведения, особенно в связи с дофаминергической (ДА-ергической) системой.

Мезолимбическая ДА-ергическая система представляет собой сложную нейронную цепь, включающую в себя прилежащее ядро (nucleus accumbens) расположенное в вентральном стриатуме, имеющее на своей поверхности рецепторы («ДА входы») из вентральной тегментальной области [11]. Данная нейронная цепь представляет собой общий нейронный путь вознаграждения. Активное высвобождение ДА в прилежащем ядре связано с реакцией вознаграждения, как на связанные с каким-либо ПАВ вознаграждением (например, кокаином), так и на «естественные» стимульные вознаграждения (секс, видеоигры) [12].

Патофизиологические модели зависимости, ориентированные на систему вознаграждения, в основном сосредоточены на обработке стимулов вознаграждения, с упором на мотивы употребления наркотических вешеств. В частности, одна из распространенных моделей гласит, что многократное употребление ПАВ, или аддиктивное поведение у людей с предрасположенностью к аддиктивной патологии, приводит «к расцвету» патологических нейронных схем, и к еще большему сдвигу поведения в гедонистический стереотип (аллостатической нагрузки) [13]. Таким образом, со временем, аддиктивное поведение «захватывает» естественную систему вознаграждения головного мозга, фактически делая ее более отзывчивой к основному ПАВ, и менее отзывчивой к другим «естественным» стимулам/вознаграждению.

Дофамин - не единственный важный нейротрансмиттер, равно как и ДА-ергическая система среднего мозга - не единственная область мозга, которая играет центральную роль в патофизиологических моделях зависимости. Нарушения, связанные с формированием аддиктивной патологии тесно связаны с функциями широкого спектра нейротрансмиттеров и нейропептидов, включая глутамат, гамма-аминомасляную кислоту (ГАМК), серотонин, норадреналин (НА), ацетилхолин, кортикотропный релизинг фактор, опиоидную систему, эндоканнабиноидную систему, окситоцин, вазопрессин и нейропептид Y [14]. В то время как ДА-ергическая система среднего мозга и связанные с ней дорзальные отделы полосатого тела в большей степени ответственны за бинджинг («заедание» проблем) и состояние интоксикации; стресс индуцированные нейрональные связи глубоких отделов амигдалы (опорное ядро терминального тяжа (BST), центральное ядро амигдалы) с центральными и периферическими отделами НА-ергической системы ответственны за формирование отдаленных последствий, связанных с употреблением ПАВ и синдрома отмены. Префронтальная кора (РFC) (орбитофронтальная, медиальная префронтальная кора, передняя поясная кора), кортикомедиальная группа ядер, инсула и парагиппокампальная извилина связаны с явлениями крейвинга.

Последние исследования позволяют дифференцировано оценить психический компонент обработки стимульных наград в системе вознаграждения. Это дает возможность анализа функций системы вознаграждения, реакций подкрепления, элементов сайленса (т.е. придать стимулам степень релевантности) и «нейрофизиологические потери» при обработке стимулов $[15,16]$. Berridge и Robinson предложили «стимуляционную» модель зависимости, которая предполагает, что «симпатия» (эмоциональная реакция удовольствия) и «желание» (стимуляционная реакция мотивации) могут быть диссоциированы нейроанатомически и нейрохимически [17].

«Синдром дефицита вознаграждения» - это еще одна нейрофизиологическая модель зависимости, ориентированная на систему вознаграждения. В основе модели лежит теория о низкой реактивности ДА-ерги- 
ческой системы среднего мозга, что приводит к формированию зависимости от различных ПАВ и аддиктивных расстройств. В результате этого формируется аддиктивное поведение, как компенсация пониженной активности ЦНС [18]. «Синдром дефицита вознаграждения» коррелирует с механизмами самолечения наркотической зависимости [19].

Различные патофизиологические модели зависимости, в целом, объясняют временные диссоциации структурных компонентов синдрома зависимости. Например, в препубертатном периоде снижение выработки ДА («дефицит вознаграждения») может привести к более раннему началу употребления наркотиков. Эпизодическое употребление наркотических веществ в подростковом возрасте может привести к химическому «возгоранию» системы вознаграждения, и быстрому формированию зависимости. Описанные патофизиологические механизмы формируют высокие риски формирования аддиктивной патологии на различных этапах развития подростков.

Мотивация - это процесс, который запускает, направляет, и поддерживает целенаправленное поведение. Мотивационные модели зависимости включают элементы мотивации, когнитивного контроля и способностей в принятии решений. Согласно мотивационным моделям аддиктивные расстройства представляют собой нарушения мотивации, при которых наибольший приоритет отдается аппетентному поведению, такому как употребление наркотических веществ, и гораздо меньше, другим видам поведения, таким как работа, школа и семья [20, 21, 22]. Таким образом, аппетентное поведение становится основной мотивацией, вытесняющей другие мотивационные стимулы.

Мотивационные модели включают в себя нейро-экономическую концепцию временного дисконтирования: выбор небольших немедленных наград, перед большими отсроченными вознаграждениями. Механизм принятия решений в данном случае связан с дискретными областями головного мозга. Патофизиологическое объяснение выбора меньших немедленных наград связан с активностью в вентральном стриатуме, и вентромедиальных отделах ПФК. Напротив, выбор больших отсроченных вознаграждений связан с дорсальными префронтальными отделами. Немедленное или отложенное вознаграждение влияет на скорость нейронального ответа [23]. Различия во временном дисконтировании можно обнаружить на разных этапах формирования зависимости, и в пределах определенных стадий, в зависимости от степени прогредиентности аддиктивной патологии. Подростки, в сравнении со взрослыми, чаще выбирают меньшую немедленную награду, в сравнении с большим отсроченным вознаграждением [24]. Взрослые и подростки с аддиктивной патологией предпочитают выбор меньших, немедленных наград, в сравнении с контрольными группами [25].

Приведенные выше данные подчеркивают важность когнитивного контроля и исполнительного функционирования в механизмах принятия решений соотношения рисков/наград. С эволютивной точки зрения развития, РFС (область головного мозга, имеющая огромное значение в механизмах реализации «нисходящего» когнитивного контроля) является одной из областей мозга, последней достигающей зрелости, и процесс часто не заканчивается до подросткового возраста. Данный фактор может способствовать формированию высокого риска возникновения аддиктивной патологии в подростковом возрасте, девиантного поведения, и других форм психических нарушений [1].

\section{Развитие головного мозга подростков и риск формирования аддиктивных расстройств}

В подростковом возрасте происходят значительные изменения в морфологии головного мозга, его архитектуре, и биохимии. Морфологические исследования показывают, что соотношение объёма серого вещества и толщины коры можно выразить в виде инвертированной параболической кривой на всем протяжении жизни, пик приходится на ранний подростковый возраст (12-14 лет), за которым следует снижение [26, 27]. Региональная морфология мозга демонстрирует временную дисперсию. На ранней стадии развития процесс созревания начинается в затылочной и сенсомоторной областях, стриатуме. PFC, а ассоциативные отделы коры достигают зрелости последними [28].

В отличие от серого вещества, в подростковом возрасте белое вещество демонстрирует более линейный рост, и увеличение нервных волокон, что приводит к достижению плато во взрослом возрасте. Недавние исследования с помощью диффузионной тензорной визуализации (DTI), позволили in vivo оценить архитектуру белого вещества, основываясь на диффузии молекул воды через нервные волокна. Фракционная анизотропия (FA) в сочетании с переменной DTI, позволяют оценить индекс организации и целостности нервных волокон. Показатели фракционной анизотропии увеличиваются в подростковом и молодом возрасте. Наиболее значимые изменения отмечаются в трактах верхнего продольного фасцикула, лучистого венца, центральной таламической лучистости, и задней ножки внутренней капсулы [29]. Параллельно с отставленным созреванием серого вещества, лобно-височные отделы белого вещества созревают на более поздних этапах развития [30, 31]. Ряд биохимических изменений, происходящих в подростковом возрасте, включают в себя изменения в ДА-ергической и ГАМК-ергической системах в период пубертатного созревания [32, 33].

С помощью различных теорий нарушения процессов онтогенеза пытаются объяснить, почему подростки демонстрируют склонность к экспериментам с наркотиками и алкоголем, и различным формам девиантного поведения. За основу предлагается принять 
дисбаланс в развитии между системами когнитивного контроля «сверху вниз», и системами стимульного вознаграждения «снизу вверх» [34]. Способность проявлять сопротивление гедонистическим стимулам в пользу проспективного осознанного поведения является одной из форм когнитивного контроля. Функции когнитивного контроля линейно совершенствуются с раннего детского возраста до взрослой поры, что связано с созреванием дорсолатеральных отделов РFС и передней поясной коры, которые являются компонентами «нисходящей» исполнительной системы. «Восходящая» подкорковая система, включая ДА-ергическую систему стриатума и среднего мозга, имеющая большое значение в формировании навыков обучения, созревает на более ранней стадии развития, чем «нисходящая» система [26]. Дисбаланс между незрелыми процессами когнитивного контроля «сверху вниз», и зрелыми (гиперактивными) «восходящими» процессами, поощрений в системе вознаграждения, в подростковом возрасте, позволяет модулирующим стимулам «вытеснить» функцию когнитивного контроля. Это приводит к повышению мотивации употребления ПАВ и аппетентному поведению.

Триумвиратная модель объясняет формирование аддиктивной патологии у подростков, и их девиантное поведение. В целом, нарушения связаны с интерфейсом трех нейрофизиологических систем: системы контроля/регуляции с вовлечением медиальных и вентральных отделов РFC; системы вознаграждения с вовлечением вентрального стриатума, и ДА-ергической системы среднего мозга; и системой угроз/опасности («снижения вреда») с вовлечением амигдалы. В данной теоретической модели неэффективная система регулирования, мощная система вознаграждения, и слабая «система снижения вреда», способствуют более активному употреблению ПАВ и другим моделям девиантного поведения [34].

Согласно другой модели развития мотивационной нейронной цепи принято разделять головной мозг на первичные и вторичные нейронные схемы мотивации. Первичные нейронные схемы включают в себя PFC, стриатум, и таламус (кортико-стриа-таламические петли), реализующие процессы принятия решений и выбор дискретного целенаправленного поведения, в том числе при различных аддикциях. Данная модель аппетентного и мотивированного поведения может быть применима как к химическим, так и нехимическим зависимостям. Первичная нейронная схема мотивации оказывает влияние на процессы мотивированного принятия решений при участии вторичной нейронной схемы мотивации, обеспечивающей связь с другими нейронными сетями головного мозга (сенсорными, аффективными, памяти, гомеостаза).

Множество факторов могут оказывать влияние на формирование риска употребления наркотических веществ и аддиктивного поведения. Факторы риска включают в себя: внутреннее состояние индивида (эмоциональный стресс) и внешние воздействия (влияние сверстников, доступность ПАВ, влияние средств массовой информации, отсутствие родительского контроля) [35].

Согласно представленной выше модели, взаимодействие областей мозга, модулирующих связь между первичными процессами компенсации нейронных схем и различными когнитивными процессами может быть нарушено у лиц с аддиктивной патологией и у подростков из группы риска. Отделы головного мозга вовлеченные в данный процесс включают в себя: амигдалу при аффективных состояниях, гиппокамп и височную кору для процессов памяти, гипоталамус для гомеостатических процессов (голод, жажда) [36], а также инсулу и теменную кору для ощущения физического и соматического благополучия и функции внимания. Таким образом, в пубертатном периоде, когда перечисленные выше дисрегуляторные нарушения достигают максимума, подростки не всегда могут регулировать свои эмоциональные состояния и мотивы поведения - наряду с определенной частью взрослых. Это объясняет ранее возникновение аддиктивной патологии и аффективных нарушений в данный возрастной период [34, 37].

До настоящего времени не утихают споры о том, влияют ли процессы формирования реакций вознаграждения и ДА-ергическая функция среднего мозга на формирование риска возникновения аддиктивной патологии и расстройств, связанных с употреблением ПАВ в подростковом возрасте. Формируют ли повышенный риск процессы гиперактивности/гипоактивности ДА-ергической системы? Некоторые исследования в этом направлении «типично» развивающихся подростков продемонстрировали повышенное формирование реакций вознаграждения в стриатуме, тогда как другие демонстрировали снижение активности реакций. Подобно взрослым с аддиктивными расстройствами, подростки, отвечающие критериям расстройств, связанных с употреблением ПАВ, и другой аддиктивной патологии, демонстрировали снижение вентральной стриальной активности во время ожидания вознаграждения, в сравнении с контрольной группой. Аналогичные модели вентральной стриальной гипоактивности коррелировали с импульсивностью и рискованным поведением у взрослых и подростков с аддиктивной патологией. Противоречия в результатах исследований подчеркивают важность изучения индивидуальных различий в подростковом возрасте. Если подростки в целом демонстрируют гиперактивность вентральной стриальной системы в процессе обработки реакций вознаграждения, то снижение стриальных реакций может выступать в роли предиктора в развитии аддиктивной патологии [38].

Рассматривая риск формирования наркотической зависимости у подростков и оценивая степень риска, следует помнить о том, что накопленные в настоящее время данные не позволяют сделать достоверные вы- 
воды о «норме» в развитии или его аберрации. При этом следует учитывать, что поведение, которое считается социально приемлемым, или нормативным в подростковом возрасте (например, экспериментирование с алкоголем, и необдуманный риск), зачастую связано с негативными последствиями и показателями неблагоприятного функционирования [39, 40]. Несмотря на то, что в некоторых культурах и обществах подобное поведение считается нормативным, оно не обходится без индивидуальных, семейных и социальных издержек. Будущие исследования должны быть направлены на изучение нейрофизиологических корреляций, которые в каждом отдельном случае помогут прогнозировать риск употребления ПАВ, и возникновения аддиктивных расстройств. Это поможет разработать целевые терапевтические вмешательства и профилактические меры для конкретных групп риска.

\section{Влияние наркотических веществ и аддиктивных расстройств на структуру и функции головного мозга подростков}

Выявление различий в структуре и функциях головного мозга подростков, особенно у тех, кто употребляет алкоголь и другие ПАВ, является не простой задачей. Нейрофизиологические изменения могут представлять собой вариант нормального развития [26], или относится к аддиктивной патологии, а в некоторых случаях отражать процессы нейроадаптации, которая представляет собой нейротоксические изменения, связанные с эпизодическим, или постоянным употреблением наркотических веществ и алкоголя, что само по себе может не отражаться на значимых нейрофизиологических процессах. Ещё большую неопределенность в исследованиях подростков с аддиктивной патологией создают различия в структуре самих исследуемых, дизайн исследования и другие переменные, такие как коморбидные психические расстройства, употребление нескольких ПАВ одновременно (полисубстантные зависимости), которые являются очень распространенным явлением среди подростков с аддиктивными расстройствами.

Лабораторные исследования демонстрируют, что мозг наиболее уязвим к воздействию ПАВ в подростковом возрасте [41]. Употребление алкоголя и каннабиноидов подростками может оказывать различное влияние на развивающийся мозг, с учетом различий, связанных с фармакологическими характеристиками ПАВ. В частности, на целостность белого вещества, и скорость выполнения когнитивных задач.

Уровень вовлеченности мозговых структур подростков с аддиктивной патологией весьма различен, и зависит от стадии заболевания: от расстройств, связанных с употреблением алкоголя (AUD), до алкогольной зависимости в течение 1-2 лет. Как правило, большинство нарушений связаны со структурным и функциональным дефицитом. Изменения отмечают- ся в белом веществе, гиппокампе, РFC, мозолистом теле, мозжечке подростков, употребляющих алкоголь и каннабис, в сравнении с контрольной группой без аддиктивных расстройств [42]. Также отмечается уменьшение объема гиппокампа у подростков с тяжелыми формами зависимости от алкоголя, в сравнении с подростками, употребляющими алкоголь и каннабис одновременно, и подростками, не употребляющими наркотики. Уменьшение объёма гиппокампа также связано с возрастом начала употребления алкоголя и длительностью заболевания. У подростков с AUD объем PFC значительно меньше, в сравнении с контрольной группой, но результаты могут варьироваться в зависимости от пола. У девочек-подростков с AUD отмечается значительно меньший объем PFC, по сравнению с девочками не употребляющих алкоголь. Подростки мужского пола с AUD имеют значительно больший объем PFC, в сравнении с мужчинами-абстинентами [42]. При этом не обнаружено никаких различий в объеме РFС подростков, употребляющих каннабис, и теми, кто его не употреблял не разу. У подростков, употребляющих каннабис, толщина червя мозжечка была значительно больше, чем в группе контроля [43]. При использовании методики DTI подростки с бинджингом алкоголя, и злоупотребляющие алкоголем, демонстрировали более низкие показатели фракционной анизотропии (FA), чем в группе контроля. Основные изменения приходились на различные участки белого вещества, включая мозолистое тело, верхний продольный пучок, лучистый венец, внутренние и внешние капсулы, ствол мозга, и корковые проекционные волокна [44, 45]. Употребление каннабиса подростками коррелировало с более низкими показателями фракционной анизотропии (FA) в области верхнего продольного пучка, постцентральной извилины, и нижнего продольного пучка, в сравнении с контрольной группой, в сравнении с подростками с бинджингом алкоголя, показатели фракционной анизотропии (FA) были увеличены [44]. Нейрокогнитивный дефицит обнаруживался у подростков, употребляющих алкоголь и каннабис одновременно. Изменения выявлялись в сфере внимания, зрительно-пространственных реакций, скорости обработки информации, памяти, функциях исполнительной системы [43]. При использовании методов f-MRT (функциональной магнитно-резонансной томографии), были выявлены изменения в пространственной рабочей (оперативной) памяти, и незначительно выраженные когнитивные нарушения, у подростков сочетано употребляющих каннабис и алкоголь, в сравнении с контрольной группой.

Структурные и функциональные изменения чаще всего возникают именно в тех областях мозга, которые отвечают за формирование значимых нейропсихологических функций (например, гиппокамп (память); PFC (исполнительные функции, планирование)). Многие из цитируемых исследований носят междисциплинарный характер, и не позволяют выявить 
причинно-следственные связи между изменениями в ЦНС и употреблением ПАВ в подростковом возрасте. Лонгитюдные исследования подростков с тщательной оценкой показателей употребления ПАВ и наличием/ отсутствием коморбидных расстройств, наряду с сочетанным употреблением нескольких психоактивных веществ, помогут выяснить влияние групповых различий на подростков из группы риска, в сравнении с последствиями, связанными с употреблением конкретных веществ. Недавние лонгитюдные исследования демонстрируют, что различия в морфологии РFС и процессов гипоактивности РFС у подростков во время ингибирования ответа системы вознаграждения могут выступать в роли предикторов более тяжелых форм употребления алкоголя и наркотиков [46].

Кроме этого, следует учитывать корреляции между стадиями заболевания и действием наркотических веществ. В подростковом возрасте, в силу анатомо-физиологических особенностей, существуют определенные «окна уязвимости», посредством которых действие ПАВ затрагивает долгосрочное функционирование. Ранний возраст начала употребления алкоголя, каннабиса, и других наркотических веществ, связан с быстрым нарастанием прогредиентности аддиктивной патологии и плохим прогнозом. Исследования на лабораторных моделях наглядно демонстрируют, что любая форма употребления ПАВ в подростковом возрасте, увеличивает в дальнейшем риск возникновения аддиктивной патологии, затрагивая систему вознаграждения и делая ее более «отзывчивой» к различным ПАВ. Употребление различных ПАВ в подростковом возрасте оказывает заметное влияние на «естественные вознаграждения», что обеспечивает связь между химическими и нехимическими (поведенческими) зависимостями.

\section{Генетика, эпигенетика, и средовые влияния}

Сложные взаимодействия генетической предрасположенности и ранних средовых влияний в подростковом возрасте предшествуют началу употребления ПАВ и аддиктивному поведению, формируя сочетание «мозг-поведение», способствующее формированию аддиктивной патологии. Влияние генов и окружающей среды может различаться в разные возрастные периоды и стадии развития аддиктивной патологии. Недавние исследования убедительно доказывают, что начало употребления ПАВ в раннем возрасте, и период «поискового наркотизма», в большинстве случаев зависят от семейных и социальных факторов. Переход от эпизодического употребления ПАВ к постоянному (компульсивному) употреблению во многом зависит от генетических факторов.

Роль генетических факторов в развитии аддиктивных расстройств значительна. Тем не менее исследования, посвященные изучению генетических факторов, влияющих на формирование аддиктивной патологии у подростков, немногочисленны. Двойные исследования демонстрируют, что генетические факторы участвуют в формировании патологии от $30 \%$ до $70 \%$ в зависимости от типа психоактивного вещества. Недавно полученные данные также доказывают роль генетических факторов при формировании поведенческих зависимостей, включая гемблинг, и интернет-зависимость, нарушения пищевого поведения в детском, и подростковом возрасте.

Исследования генома связаны с множеством полиморфизмов генов при аддиктивной патологии, однако, исследования подростков с химической и нехимической зависимостью отсутствуют [47]. Аддиктивные расстройства у взрослых связаны с генами и генетическими локусами и различными нейробиологическими процессами: функцией транспорта нейромедиаторов/нейропептидов (серотонина, ДА, НА, транспортеров ДА рецепторов 2, мю-опиоидных рецепторов), веществами, влияющими на метаболизм (цитохром Р450 2А6, ДА-бета-гидроксилазы), факторами роста (нейротрофический фактор головного мозга), и вторичными мессенджеров. Недавно проведенное исследование продемонстрировало, что полиморфизм гена, кодирующего мю-опиоидные рецепторы, связан с злоупотреблением алкоголем в подростковом возрасте [48]. Генетическая предрасположенность $\mathrm{K}$ аддиктивной патологии может коррелировать с генетической уязвимостью к определенному типу ПАВ.

В последнее десятилетие в эпигенетике исследовались взаимодействия генов и средовых влияний $(\mathrm{G} \times \mathrm{E})$. В частности, изучалось, как экспрессия общих генетических вариантов и условий окружающей среды в раннем детстве может влиять на развитие аддиктивной патологии. Понимание процессов взаимодействия $\mathrm{G} \times \mathrm{E}$, в частности, как взаимодействуют «гены» и «воспитание/среда», поможет в изучении аддиктивных расстройств и других форм психической патологии. Недавнее исследование $\mathrm{G} \times$ Е обнаружило взаимодействие между вариантом гена, кодирующего рецепторы кортикотропин-релизинг гормона 1 и стрессовыми событиями в жизни, влияющими на возраст начала употребления алкоголя и прогредиентность алкоголизации у подростков [49].

Проспективные исследования $\mathrm{G} \times \mathrm{E}$ должны быть направлены на изучение влияния подростковой наркотизации и алкоголизации, на формирование аддиктивной патологии у взрослых и коморбидных психических расстройств. Так, функциональный полиморфизм гена, кодирующего катехол-О-метилтрансферазу, связан с употреблением каннабиса в подростковом возрасте, и развитием психотических расстройств во взрослом периоде. При этом наличие аллели, кодирующей валин, повышает риск возникновения данных патологий [50]. В рамках проспективных исследований должны быть четко определены временные факторы воздействия средовых влияний - с целью оценки степени риска, особенно в контексте динамических изменений головного мозга, происходящих в детском и подростковом периодах. 


\section{Возможности профилактики и перспективы терапевтического вмешательства}

Знание нейрофизиологических механизмов аддиктивных расстройств в подростковом возрасте открывает перспективы для улучшения мер профилактики и лечения аддиктивной патологии, а также возможности изменений в текущей наркоситуации. Накопленные знания могут помочь в разработке новых препаратов, которые будут нацелены на точки-мишени нейромедиаторных систем. Одним из перспективных направлений является генная терапия и создание лекарственных вакцин [51].

Лекарственные средства, основным механизмом которых является блокада высвобождения ДА, демонстрируют крайне низкую эффективность, а в ряде случаев приводят к утяжелению аддиктивных расстройств, в частности нехимических форм аддиктивного поведения [51, 52]. Всё больший интерес вызывают лекарственные агенты, которые оказывают модулирующее влияние на передачу ДА в системе вознаграждения посредством глутаматергических (N-ацетилцистеин; «Акампросат») и опиоидных («Налтрексон», «Бупренорфин») рецепторных систем [51, 53]. Лишь некоторые из упомянутых выше препаратов применялись для лечения аддиктивной патологии у подростков. Поэтому необходимы дальнейшие исследования с целью изучения безопасности и эффективности препаратов в подростковом возрасте [53].

Нельзя оставлять без внимания коморбидные психические расстройства и лекарственное взаимодействие при одновременном лечении коморбидных психических расстройств и аддиктивной патологии. Данные факторы могут влиять на прогредиентность аддиктивных расстройств и продуктивную симптоматику коморбидных заболеваний. Клинические исследования коморбидных заболеваний для расстройств, связанных с употреблением ПАВ (SUD), в частности большого депрессивного расстройства (MDD/БДР) и синдрома дефицита внимания/гиперактивности (СДВГ), свидетельствуют о том, что послабление основных симптомов коморбидной патологии связано с сокращением употребления наркотических веществ.

Выявление общих нейрофизиологических закономерностей развития аддиктивной патологии в подростковом возрасте может стать еще одним перспективным направлением поиска новых психофармакологических методов терапии [54]. При разработке стратегий профилактики и терапевтического вмешательства должны учитываться индивидуальные биологические факторы, оказавшие влияние на формирование аддиктивной патологии, а также «сильные» свойства личности подростков.

Подростки с физиологической точки зрения более восприимчивы к «вознаграждениям» и меньше реагируют на отрицательные стимулы, в сравнении со взрослыми. Поэтому программы реабилитации и ресоциализации должны использовать механизмы положительного подкрепления, а не наказания, или ме- тодик, основанных на отрицательном подкреплении, что может стать эффективным инструментом [55]. Вместо попыток устранить «стимульное» отклоняющееся поведение и ограничения доступа к девиантным микросоциальным группам, необходимо разрешить подросткам доступ к увеселительным мероприятиям в контролируемых условиях, что может существенно снизить риск возникновения рецидива аддиктивной патологии.

Ресоциализация подростков с аддиктивной патологией, наряду с возможностью быстрого реагирования на нештатные ситуации, основанные на методах положительного подкрепления (вознаграждения) просоциального поведения, способствует существенному снижению вовлеченности подростков в наркопотребление. Восстановление когнитивного контроля с помощью когнитивно-поведенческой терапии оказалось эффективным инструментом терапии аддиктивных расстройств у подростков [56, 57]. Данные предварительных исследований свидетельствуют о том, что эффекты когнитивной терапии связаны с изменением функциональной активности нейронных сетей, ответственных за реализацию мотивационной составляющей и когнитивного контроля [58].

Эпидемиологические и клинические данные свидетельствуют о том, что девочки-подростки, в сравнении с юношами, отличаются различными механизмами реализации факторов защиты, и индивидуальными профилями риска возникновения аддиктивной патологии. Это формирует большую вероятность употребления наркотических веществ, чем у юношей. Исследование роли гендерных аспектов при терапевтических вмешательствах и развитие программ гендерно-центрированной терапии может улучшить результаты терапии в подростковой среде.

Обсуждение и принятие законодательных актов в области наркополитики необходимо сопровождать консультациями со специалистами в области психического здоровья и аддиктивных расстройств. Налоговое законодательство в области табачных изделий и алкогольной продукции должно выступать в роли эффективного инструмента для мер, направленных на снижение употребления табака и алкоголя, как для взрослых граждан, так и для подростков [59]. Подростки являются «гиперпотребителями» цифрового контента, распространяемого через средства массовой информации. Понимание механизмов влияния рекламы/маркетинговых стратегий «гедонистических продуктов» (алкоголя, табака, шоколада, газированных напитков) на развитие у подростков аддиктивных расстройств поможет в более глубоком изучении различных форм аппетентного поведения.

\section{Заключение}

Аддиктивные расстройства включают в себя как химические зависимости, так и поведенческие аддикции. 
На сегодняшний день аддиктивные расстройства остаются одними из самых дорогостоящих заболеваний для общества. Подростковый возраст является критическим периодом в развитии любого человека $[60,61]$, и именно поэтому необходимо уделять особое внимание аддиктивной патологии в подростковом периоде с целью защиты будущих поколений и ограничения потенциальных социальных издержек. Новые данные о нейрофизиологии аддиктивных расстройств и потенциальных рисках возникновения наркотической зависимости могут способствовать прогрессу в области подростковой аддиктологии, повышая эффективность профилактических мер и терапевтических вмешательств.

\section{Список литературы:}

1. Viner R.M., Ozer E.M., Denny S., Marmot M., Resnick M., Fatusi A., Currie C. Adolescence and the social determinants of health. Lancet. 2012; 379(9826): 1641-1652. DOI: 10.1016/S01406736(12)60149-4

2. Casey B.J., Jones R.M., Hare T.A. The adolescent brain. Ann. N.Y. Acad. Sci. 2008; 1124: 111-1126. DOI: 10.1196/annals.1440.010

3. Eaton D.K., Kann L., Kinchen S., Shanklin S., Ross J., Hawkins J., Harris W.A., Lowry R., McManus T., Chyen D., Lim C., Brener N.D., Wechsler H.; Centers for Disease Control and Prevention (CDC). Youth risk behavior surveillance--United States, 2007. MMWR Surveill. Summ. 2008; 57(4): 1-131.

4. Johnston L.D., O'Malley P.M., Bachman J.G., Schulenberg J.E. Monitoring the future: National Survey Results on drug use, 1975-2008. Volume II: college students and adults ages 19-50. (NIH Publication No. 09-7403). Bethesda, MD: National Institute on Drug Abuse, 2009. $329 \mathrm{p}$.

5. Grant J.E., Potenza M.N., Weinstein A., Gorelick D.A. Introduction to behavioral addictions. Am. J. Drug Alcohol Abuse. 2010; 36(5): 233241. DOI: $10.3109 / 00952990.2010 .491884$

6. Maddux J.F., Desmon D.P. Addiction or dependence? Addiction. 2000; 95(5): 661-665. DOI: DOI: 10.1046/j.13600443.2000.9556611.x

7. Koob G. F., Volkow N. D. Neurocircuitry of addiction. Neuropsycopharmacology. 2010; 35: 217-238. DOI: 10.1038/npp.2009.110

8. Heo J., Oh J., Subramanian S.V., Kim Y., Kawachi I. Addictive internet use among Korean adolescents: a national survey. PloS One. 2014; 9(2): e87819. DOI: 10.1371/journal.pone.0087819

9. Desai R.A., Krishnan-Sarin S., Cavallo D., Potenza M.N. Videogaming among high school students: health correlates, gender differences, and problematic gaming. Pediatrics. 2010; 1414-1424. DOI: $10.1542 /$ peds. 2009-2706

10. Liu T.C., Desai R.A., Krishnan-Sarin S., Cavallo D.A., Potenza M.N. Problematic Internet use and health in adolescents: data from a high school survey in Connecticut. J. Clin. Psychiatry. 2011; 72(6): 836-845. DOI: 10.4088/JCP.10m06057

11. Grant J.E., Potenza M.N., Krishnan-Sarin S., Cavallo D.A., Desai R.A. Shopping problems among high school students. Compr. Psychiatry. 2011; 52(3): 247-252. DOI: $10.1016 / \mathrm{j}$. comppsych.2010.06.006

12. Volkow N.D., Wang G.J., Baler R.D. Reward, dopamine and the control of food intake: implications for obesity. Trend Cogn. Sci. 2011; 15(1): 37-46. DOI: 10.1016/j.tics.2010.11.001

13. Yip S.W., Desai R.A., Steinberg M.A., Rugle L., Cavallo D.A., Krishnan-Sarin S., Potenza M.N. Health/functioning characteristics, gambling behaviors, and gambling-related motivations in adolescents stratified by gambling problem severity: Findings from a high school survey. Am. J. Addict. 2011; 20(6): 495-508. DOI: 10.1111/j.15210391.2011.00180.x

14. Cooper Z.D. Adverse effects of synthetic cannabinoids: management of acute toxicity and withdrawal. Curr. Psychiatry Rep. 2016; 18(5): 52. DOI: $10.1007 / \mathrm{s} 11920-016-0694-1$

15. Sulzer D. How addictive drugs disrupt presynaptic dopamine neurotransmission. Neuron. 2011; 69(4): 628-649. DOI: 10.1016/j.neuron.2011.02.010
16. Kenny P.J. Reward mechanisms in obesity: new insights and future directions. Neuron. 2011; 69(4): 664-679. DOI: 10.1016/j.neuron.2011.02.016

17. Berridge K.C., Robinson T.E., Aldridge J.W. Dissecting components of reward: 'liking', 'wanting', and learning. Curr Opin Pharmacol. 2009; 9(1): 65-73. DOI: 10.1016/j.coph.2008.12.014.

18. George O., Le Moal M., Koob G.F. Allostasis and addiction: role of the dopamine and corticotropin-releasing factor systems. Physiol. Behav. 2012; 106(1): 58-64. DOI: 10.1016/j.physbeh.2011.11.004

19. The Neurobiology of addiction: new vistas. Ed.: Robbins T.W., Everitt B.J., Nutt D.J. New York: Oxford University Press, 2010. 318 p.

20. Schultz W. Potential vulnerabilities of neuronal reward, risk, and decision mechanisms to addictive drugs. Neuron. 2011; 69(4): 603-617. DOI: 10.1016/j.neuron.2011.02.014

21. Volkow N.D., Li T.K. Drug addiction: the neurobiology of behavior gone awry. Nat. Rev. Neurosci. 2004; 5(12): 963-970. DOI: 10.1038/nrn1539

22. Борукаева И.Х., Абазова 3.Х., Кумыков В.К. Влияние кратковременной гипоксии на биоэлектрическую активность головного мозга детей, подростков и юношей. Фундаментальные исследования. 2014; 4(3): 466-471.

23. Argamany J.R., Reveles K.R., Duhon B. Synthetic cannabinoid hyperemesis resulting in rhabdomyolysis and acute renal failure. Am. J. Emerg. Med. 2016; 34(4): 765. DOI: 10.1016/j.ajem.2015.08.051

24. Khantzian E.J., Mack J.E., Schatzberg A.F. Heroin use as an attempt to cope: Clinical observations. Am. J. Psychiatry. 1974; 131(2): 160164. DOI: 10.1176/ajp.131.2.160

25. Dalley J.W., Everitt B.J., Robbins T.W. Impulsivity, compulsivity, and top-down cognitive control. Neuron. 2011; 69(4): 680-694. DOI: 10.1016/j.neuron.2011.01.020

26. Monitoring the Future Study: Trends in Prevalence of Various Drugs. Available at: https://www.drugabuse.gov/trends-statistics/monitoring-future/monitoring-future-study-trends-in-prevalence-various-drugs Retrieved: 07.04.2019

27. Stanger C., Elton A., Ryan S.R., James G.A., Budney A.J., Kilts C.D. Neuroeconomics and adolescent substance abuse: individual differences in neural networks and delay discounting. J. Am. Acad. Child Adolesc. Psychiatry. 2013; 52(7): 747-755. e6. DOI: 10.1016/j.jaac.2013.04.013

28. Kane E.M., Hinson J.S., Jordan C.D., Paziana K., Sauber N.J., Rothman R.E., Stolbach A.I. Bradycardia and hypotension after synthetic cannabinoid use: a case series. Am. J. Emerg. Med. 2016; 34(10): 2055. e1-2055.e2. DOI: 10.1016/j.ajem.2016.03.007

29. Gogtay N., Giedd J.N., Lusk L., Hayashi K.M., Greenstein D., Vaituzis A.C., Nugent T.F. 3rd, Herman D.H., Clasen L.S., Toga A.W., Rapoport J.L., Thompson P.M. Dynamic mapping of human cortical development during childhood through early adulthood. Proc. Natl. Acad .Sci USA. 2004; 101(21): 8174-8179. DOI: 10.1073/ pnas. 0402680101

30. Aoun E. G., Christopher P. P., Ingraham J. W. Emerging drugs of abuse: clinical and legal considerations. R. I. Med. J. 2014; 97(6): 41-45.

31. Tamnes C.K., Ostby Y., Fjell A.M., Westlye L.T., Due-Tønnessen P., Walhovd K.B. Brain maturation in adolescence and young adulthood: regional age-related changes in cortical thickness and white matter volume and microstructure. Cereb. Cortex. 2009; 20(3): 534-548. DOI: 10.1093/cercor/bhp118

32. Shaw P., Kabani N.J., Lerch J.P., Eckstrand K., Lenroot R., Gogtay N., Greenstein D., Clasen L., Evans A., Rapoport J.L., Giedd J.N., Wise S.P. Neurodevelopmental trajectories of the human cerebral cortex. J. Neurosci. 2008; 28(14): 3586-3594. DOI: 10.1523/ JNEUROSCI.5309-07.2008

33. Atik S.U., Dedeoğlu R., Varol F., Çam H., Eroğlu A.G., Saltık L. Cardiovascular side effects related with use of synthetic cannabinoids "bonzai": two case reports. Turk. Pediatri. Ars. 2015; 50(1): 61-64. DOI: $10.5152 /$ tpa.2015.2609

34. Giedd J.N. The teen brain: insights from neuroimaging. J. Adolesc. Health. 2008; 42(4): 335-343. DOI: 10.1016/j.jadohealth

35. Елистратова И.В. Роль стресса в обострении атопического дерматита у взрослых людей. Патогенез. 2018; 16(4): 157-160. DOI: $10.25557 / 2310-0435.2018 .04 .157-160$

36. Bava S., Jacobus J., Mahmood O., Yang T.T., Tapert S.F. Neurocognitive correlates of white matter quality in adolescent substance users. Brain Cogn. 2010; 72(3): 347-354. DOI: 10.1016/j.bandc.2009.10.012

37. Dahl R.E., Gunnar M.R. Heightened stress responsiveness and emotional reactivity during pubertal maturation: implications for psychopathology. Dev. Psychopathol. 2009; 21(1): 1-6. DOI: 10.1017/ S0954579409000017 
38. Metcalfe J., Mischel W. A hot/cool-system analysis of delay of gratification: dynamics of willpower. Psychol. Rev. 1999; 106(1): 3-19. DOI: 10.1037/0033-295x.106.1.3

39. Belujon P., Grace A.A. Hippocampus, amygdala, and stress: interacting systems that affect susceptibility to addiction. Ann. N.Y. Acad. Sci. 2011; 1216: 114-121. DOI: 10.1111/j.1749-6632.2010.05896.x

40. Davidson S., Lear M., Shanley L., Hing B., Baizan-Edge A., Herwig A., Quinn J.P., Breen G., McGuffin P., Starkey A., Barrett P. MacKenzie A. Differential activity by polymorphic variants of a remote enhancer that supports galanin expression in the hypothalamus and amygdala: implications for obesity, depression and alcoholism. Neuropsychopharmacology. 2011; 36(11): 2211-2221. DOI: 10.1038/ npp. 2011.93

41. Volkow N.D., Swanson J.M. Variables that affect the clinical use and abuse of methylphenidate in the treatment of ADHD. Am. J. Psychiatry. 2003; 160(11): 1909-1918. DOI: 10.1176/appi.ajp.160.11.1909

42. Somerville L.H., Hare T., Casey B.J. Frontostriatal maturation predicts cognitive control failure to appetitive cues in adolescents. J. Cogn. Neurosci. 2011; 23(9): 2123-2134. DOI: 10.1162/jocn.2010.21572

43. Balodis I.M., Kober H., Worhunsky P.D., Stevens M.C., Pearlson G.D., Potenza M.N. Diminished frontostriatal activity during processing of monetary rewards and losses in pathological gambling. Biol. Psychiatry. 2012; 71(8): 749-757. DOI: 10.1016/j.biopsych.2012.01.006

44. Schneider S., Peters J., Bromberg U., Brassen S., Miedl S.F., Banaschewski T., Barker G.J., Conrod P., Flor H., Garavan H., Heinz A., Ittermann B., Lathrop M., Loth E., Mann K., Martinot J.L., Nees F., Paus T., Rietschel M., Robbins T.W., Smolka M.N., Spanagel R., Ströhle A., Struve M., Schumann G., Büchel C.; IMAGEN Consortium. Risk taking and the adolescent reward system: a potential common link to substance abuse. Am. J. Psychiatry. 2012; 169(1): 39-46. DOI: 10.1176/appi.ajp.2011.11030489

45. Rao H., Mamikonyan E., Detre J.A., Siderowf A.D., Stern M.B., Potenza M.N., Weintraub D. Decreased ventral striatal activity with impulse control disorders in Parkinson's disease. Mov. Disord. 2010; 25(11): 1660-1669. DOI: $10.1002 /$ mds. 23147

46. Eiden L.E., Weihe E. VMAT2: a dynamic regulator of brain monoaminergic neuronal function interacting with drugs of abuse. Ann. N.Y. Acad. Sci. 2011; 1216: 86-98. DOI: 10.1111/j.17496632.2010.05906.x

47. Магаева С.В., Морозов С.Г. Нейроиммунофизиология. Москва: Изд-во ГУ НИИ биомедицинской химии им. В Н. Ореховича PAMH, 2005. $160 \mathrm{c}$

48. Шагиахметов Ф. Ш., Шамакина И. Ю., Давыдова Т.В. Механизмы контроля потребления алкоголя: экспрессия генов ноцицепин/каппа-опиоидной системы в мозге крыс. Патогенез. 2018; 16(4): 112-114. DOI: 10.25557/2310-0435.2018.04.112-114.

49. Norman A.L., Pulido C., Squeglia L.M., Spadoni A.D., Paulus M.P., Tapert S.F. Neural activation during inhibition predicts initiation of substance use in adolescence. Drug Alcohol Depend. 2011; 119(3): 216223. DOI: 10.1016/j.drugalcdep.2011.06.019

50. Mahmood O.M., Goldenberg D., Thayer R., Migliorini R., Simmons A.N., Tapert S.F. Adolescents' fMRI activation to a response inhibition task predicts future substance use. Addict. Behav. 2013; 38(1): 1435-1441. DOI: 10.1016/j.addbeh.2012.07.012

51. Rando K., Chaplin T.M., Potenza M.N., Mayes L., Sinha R. Prenatal cocaine exposure and gray matter volume in adolescent boys and girls: relationship to substance use initiation. Biol. Psychiatry. 2013; 74(7): 482-489. DOI: 10.1016/j.biopsych.2013.04.030

52. White C.M. The pharmacologic and clinical effects of illicit synthetic cannabinoids. .J Clin. Pharmacol. 2017; 57(3): 297-304. DOI: $10.1002 /$ jcph. 827

53. Lisdahl K.M., Gilbart E.R., Wright N.E., Shollenbarger S. Dare to delay? The impacts of adolescent alcohol and marijuana use onset on cognition, brain structure, and function. Front Psychiatry. 2013; 4: 53. DOI: $10.3389 /$ fpsyt.2013.00053

54. Hammond C. J., Mayes L. C., Potenza M. N. Neurobiology of adolescent substance use and addictive behaviors: prevention and treatment implications. Adolesc. Med. State Art. Rev. 2014; 25(1): 15-32.

55. Montag C., Kirsch P., Sauer C., Markett S., Reuter M. The role of the CHRNA4 gene in Internet addiction: a case-control study. J. Addict. Med. 2012; 6(3): 191-195. DOI: 10.1097/ADM.0b013e31825ba7e7

56. Potenza M.N., Sofuoglu M., Carroll K.M., Rounsaville B.J. Neuroscience of behavioral and pharmacological treatments for addictions. Neuron. 2011; 69(4): 695-712. DOI: 10.1016/j.neuron.2011.02.009
57. Miliano C., Serpelloni G., Rimondo C., Mereu M., Marti M., De Luca M.A. Neuropharmacology of new psychoactive substances (NPS): focus on the rewarding and reinforcing properties of cannabimimetics and amphetamine-like stimulants. Front. Neurosci. 2016; 10: 153. DOI: 10.3389/fnins.2016.00153

58. Cha H.J., Seong Y.H., Song M.J., Jeong H.S., Shin J., Yun J., Han K., Kim Y.H., Kang H., Kim H.S.Neurotoxicity of synthetic cannabinoids JWH-081 and JWH-210. Biomol. Ther. (Seoul). 2015; 23(6): 597-603. DOI: 10.4062/biomolther.2015.057

59. Karila L., Benyamina A. Synthetic Cannabinoid Use. In: Cannabis Use Disorders. Ed: Montoya I.D., Weiss S.R.B. Springer, Cham, 2019. 163-176 p. DOI: 10.1007/978-3-319-90365-1

60. Акмаев Э.Г., Александров А.С., Алчинова И.Б., Бочаров Е.В., Карганов М.Ю., Крыжановский Г.Н., Кучеряну В.Г., Магаева С.В., Морозов С.Г., Носкин Л.А., Панфилов Д.Н., Пшенникова М.Г., Сарманаев С.Х., Сепиашвили Р.И., Сюч Н.И., Фисун А.Я., Чувин Б.Т. Санология. Под ред.: Кубатиева А.А., Симоненко В.Б. М.: Наука, 2014. 285 с.

61. Актуальные проблемы нейроиммунопатологии. Под ред.: Крыжановского Г.Н., Магаевой С.В., Морозова С.Г. М.: Гениус Медиа, 2012. 424 с.

\section{References}

1. Viner R.M., Ozer E.M., Denny S., Marmot M., Resnick M., Fatusi A., Currie C. Adolescence and the social determinants of health. Lancet. 2012; 379(9826): 1641-1652. DOI: 10.1016/S01406736(12)60149-4

2. Casey B.J., Jones R.M., Hare T.A. The adolescent brain. Ann. N.Y. Acad. Sci. 2008; 1124: 111-1126. DOI: 10.1196/annals.1440.010

3. Eaton D.K., Kann L., Kinchen S., Shanklin S., Ross J., Hawkins J., Harris W.A., Lowry R., McManus T., Chyen D., Lim C., Brener N.D., Wechsler H.; Centers for Disease Control and Prevention (CDC). Youth risk behavior surveillance--United States, 2007. MMWR Surveill. Summ. 2008; 57(4): 1-131.

4. Johnston L.D., O’Malley P.M., Bachman J.G., Schulenberg J.E. Monitoring the future: National Survey Results on drug use, 1975-2008. Volume II: college students and adults ages 19-50. (NIH Publication No. 097403). Bethesda, MD: National Institute on Drug Abuse, 2009. 329 p.

5. Grant J.E., Potenza M.N., Weinstein A., Gorelick D.A. Introduction to behavioral addictions. Am. J. Drug Alcohol Abuse. 2010; 36(5): 233 241. DOI: $10.3109 / 00952990.2010 .491884$

6. Maddux J.F., Desmon D.P.Addiction or dependence? Addiction. 2000; 95(5): 661-665. DOI: DOI: 10.1046/j.1360-0443.2000.9556611.x

7. Koob G. F., Volkow N. D. Neurocircuitry of addiction. Neuropsycopharmacology. 2010; 35: 217-238. DOI: 10.1038/npp.2009.110

8. Heo J., Oh J., Subramanian S.V., Kim Y., Kawachi I. Addictive internet use among Korean adolescents: a national survey. PloS One. 2014; 9(2): e87819. DOI: 10.1371/journal.pone.0087819

9. Desai R.A., Krishnan-Sarin S., Cavallo D., Potenza M.N. Video-gaming among high school students: health correlates, gender differences, and problematic gaming. Pediatrics. 2010; 1414-1424. DOI: 10.1542/peds.2009-2706

10. Liu T.C., Desai R.A., Krishnan-Sarin S., Cavallo D.A., Potenza M.N. Problematic Internet use and health in adolescents: data from a high school survey in Connecticut. J. Clin. Psychiatry. 2011; 72(6): 836-845. DOI: $10.4088 / J C P .10 m 06057$

11. Grant J.E., Potenza M.N., Krishnan-Sarin S., Cavallo D.A., Desai R.A. Shopping problems among high school students. Compr. Psychiatry. 2011; 52(3): 247-252. DOI: 10.1016/j.comppsych.2010.06.006

12. Volkow N.D., Wang G.J., Baler R.D. Reward, dopamine and the control of food intake: implications for obesity. Trend Cogn. Sci. 2011; 15(1): 37-46. DOI: 10.1016/j.tics.2010.11.001

13. Yip S.W., Desai R.A., Steinberg M.A., Rugle L., Cavallo D.A., Krishnan-Sarin S., Potenza M.N. Health/functioning characteristics, gambling behaviors, and gambling-related motivations in adolescents stratified by gambling problem severity: Findings from a high school survey. Am. J. Addict. 2011; 20(6): 495-508. DOI: 10.1111/j.15210391.2011.00180.x

14. Cooper Z.D. Adverse effects of synthetic cannabinoids: management of acute toxicity and withdrawal. Curr. Psychiatry Rep. 2016; 18(5): 52. DOI: $10.1007 / \mathrm{s} 11920-016-0694-1$

15. Sulzer D. How addictive drugs disrupt presynaptic dopamine neurotransmission. Neuron. 2011; 69(4): 628-649. DOI: 10.1016/j.neuron.2011.02.010 
16. Kenny P.J. Reward mechanisms in obesity: new insights and future directions. Neuron. 2011; 69(4): 664-679. DOI: 10.1016/j.neuron.2011.02.016

17. Berridge K.C., Robinson T.E., Aldridge J.W. Dissecting components of reward: 'liking', 'wanting', and learning. Curr Opin Pharmacol. 2009; 9(1): 65-73. DOI: 10.1016/j.coph.2008.12.014.

18. George O., Le Moal M., Koob G.F. Allostasis and addiction: role of the dopamine and corticotropin-releasing factor systems. Physiol. Behav. 2012; 106(1): 58-64. DOI: 10.1016/j.physbeh.2011.11.004

19. The Neurobiology of addiction: new vistas. Ed.: Robbins T.W., Everitt B.J., Nutt D.J. New York: Oxford University Press, 2010. 318 p.

20. Schultz W. Potential vulnerabilities of neuronal reward, risk, and decision mechanisms to addictive drugs. Neuron. 2011; 69(4): 603-617. DOI: 10.1016/j.neuron.2011.02.014

21. Volkow N.D., Li T.K. Drug addiction: the neurobiology of behaviorgone awry. Nat. Rev. Neurosci. 2004; 5(12): 963-970. DOI: 10.1038/nrn1539

22. Borukaeva I.Kh., Abazova Z.H., Kumykov V.K. [The effect of intermitted hypoxia on bioelectric activity of the brain in children, adolescents and youth]. Fundamental'nye issledovaniya. [Basic research]. 2015; 4 (3): 466-471. (in Russian)

23. Argamany J.R., Reveles K.R., Duhon B. Synthetic cannabinoid hyperemesis resulting in rhabdomyolysis and acute renal failure. Am. J. Emerg. Med. 2016; 34(4): 765. DOI: 10.1016/j.ajem.2015.08.051

24. Khantzian E.J., Mack J.E., Schatzberg A.F. Heroin use as an attempt to cope: Clinical observations. Am. J. Psychiatry. 1974; 131(2): 160164. DOI: 10.1176/ajp.131.2.160

25. Dalley J.W., Everitt B.J., Robbins T.W. Impulsivity, compulsivity, and top-down cognitive control. Neuron. 2011; 69(4): 680-694. DOI: 10.1016/j.neuron.2011.01.020

26. Monitoring the Future Study: Trends in Prevalence of Various Drugs. Available at: https://www.drugabuse.gov/trends-statistics/monitoring-future/monitoring-future-study-trends-in-prevalence-various-drugs Retrieved: 07.04.2019

27. Stanger C., Elton A., Ryan S.R., James G.A., Budney A.J., Kilts C.D. Neuroeconomics and adolescent substance abuse: individual differences in neural networks and delay discounting. J. Am. Acad. Child Adolesc. Psychiatry. 2013; 52(7): 747-755. e6. DOI: 10.1016/j. jaac.2013.04.013

28. Kane E.M., Hinson J.S., Jordan C.D., Paziana K., Sauber N.J., Rothman R.E., Stolbach A.I. Bradycardia and hypotension after synthetic cannabinoid use: a case series. Am. J. Emerg. Med. 2016; 34(10): 2055. e1-2055.e2. DOI: 10.1016/j.ajem.2016.03.007

29. Gogtay N., Giedd J.N., Lusk L., Hayashi K.M., Greenstein D., Vaituzis A.C., Nugent T.F. 3rd, Herman D.H., Clasen L.S., Toga A.W., Rapoport J.L., Thompson P.M. Dynamic mapping of human cortical development during childhood through early adulthood. Proc. Natl. Acad. Sci USA. 2004; 101(21): 8174-8179. DOI: 10.1073/pnas.0402680101

30. Aoun E. G., Christopher P. P., Ingraham J. W. Emerging drugs of abuse: clinical and legal considerations. R. I. Med. J. 2014; 97(6): 41-45.

31. Tamnes C.K., Ostby Y., Fjell A.M., Westlye L.T., Due-Tønnessen P., Walhovd K.B. Brain maturation in adolescence and young adulthood: regional age-related changes in cortical thickness and white matter volume and microstructure. Cereb. Cortex. 2009; 20(3): 534548. DOI: $10.1093 /$ cercor/bhp 118

32. Shaw P., Kabani N.J., Lerch J.P., Eckstrand K., Lenroot R., Gogtay N., Greenstein D., Clasen L., Evans A., Rapoport J.L., Giedd J.N., Wise S.P. Neurodevelopmental trajectories of the human cerebral cortex. J. Neurosci. 2008; 28(14): 3586-3594. DOI: 10.1523/JNEUROSCI.5309-07.2008

33. Atik S.U., Dedeoğlu R., Varol F., Çam H., Eroğlu A.G., Saltık L. Cardiovascular side effects related with use of synthetic cannabinoids "bonzai": two case reports. Turk. Pediatri. Ars. 2015; 50(1): 61-64. DOI: $10.5152 /$ tpa.2015.2609

34. Giedd J.N. The teen brain: insights from neuroimaging. J. Adolesc. Health. 2008; 42(4): 335-343. DOI: 10.1016/j.jadohealth

35. Elistratova I.V. [Involvement of stress in relapse of atopic dermatitis in adults]. Patogenez. [Pathogenesis]. 2018; 16(4): 157-160. DOI: 10.25557/2310-0435.2018.04.157-160 (in Russian)

36. Bava S., Jacobus J., Mahmood O., Yang T.T., Tapert S.F. Neurocognitive correlates of white matter quality in adolescent substance users Brain Cogn. 2010; 72(3): 347-354. DOI: 10.1016/j.bandc.2009.10.012

37. Dahl R.E., Gunnar M.R. Heightened stress responsiveness and emotional reactivity during pubertal maturation: implications for psychopathology. Dev. Psychopathol. 2009; 21(1): 1-6. DOI: 10.1017/ S0954579409000017
38. Metcalfe J., Mischel W. A hot/cool-system analysis of delay of gratification: dynamics of willpower. Psychol. Rev. 1999; 106(1): 3-19. DOI: 10.1037/0033-295x.106.1.3

39. Belujon P., Grace A.A. Hippocampus, amygdala, and stress: interacting systems that affect susceptibility to addiction. Ann. N.Y. Acad Sci. 2011; 1216: 114-121. DOI: 10.1111/j.1749-6632.2010.05896.x

40. Davidson S., Lear M., Shanley L., Hing B., Baizan-Edge A., Herwig A. Quinn J.P., Breen G., McGuffin P., Starkey A., Barrett P., MacKenzie A. Differential activity by polymorphic variants of a remote enhancer that supports galanin expression in the hypothalamus and amygdala: implications for obesity, depression and alcoholism. Neuropsychopharmacology. 2011; 36(11): 2211-2221. DOI: 10.1038/npp.2011.93

41. Volkow N.D., Swanson J.M. Variables that affect the clinical use and abuse of methylphenidate in the treatment of ADHD. Am. J. Psychiatry. 2003; 160(11): 1909-1918. DOI: 10.1176/appi.ajp.160.11.1909

42. Somerville L.H., Hare T., Casey B.J. Frontostriatal maturation predicts cognitive control failure to appetitive cues in adolescents. J. Cogn. Neurosci. 2011; 23(9): 2123-2134. DOI: 10.1162/jocn.2010.21572

43. Balodis I.M., Kober H., Worhunsky P.D., Stevens M.C., Pearlson G.D., Potenza M.N. Diminished frontostriatal activity during processing of monetary rewards and losses in pathological gambling. Biol. Psychiatry. 2012; 71(8): 749-757. DOI: 10.1016/j.biopsych.2012.01.006

44. Schneider S., Peters J., Bromberg U., Brassen S., Miedl S.F., Banaschewski T., Barker G.J., Conrod P., Flor H., Garavan H., Heinz A., Ittermann B., Lathrop M., Loth E., Mann K., Martinot J.L., Nees F., Paus T., Rietschel M., Robbins T.W., Smolka M.N., Spanagel R., Ströhle A., Struve M., Schumann G., Büchel C.; IMAGEN Consortium. Risk taking and the adolescent reward system: a potential common link to substance abuse. Am. J. Psychiatry. 2012; 169(1): 39-46. DOI: 10.1176/appi.ajp.2011.11030489

45. Rao H., Mamikonyan E., Detre J.A., Siderowf A.D., Stern M.B., Potenza M.N., Weintraub D. Decreased ventral striatal activity with impulse control disorders in Parkinson's disease. Mov. Disord. 2010; 25(11): 1660-1669. DOI: $10.1002 / \mathrm{mds} .23147$

46. Eiden L.E., Weihe E. VMAT2: a dynamic regulator of brain monoaminergic neuronal function interacting with drugs of abuse. Ann. N.Y. Acad. Sci. 2011; 1216: 86-98. DOI: 10.1111/j.1749-6632.2010.05906.x

47. Magaeva S.V., Morozov S.G. [Neuroimmunophysiology]. Moscow: Publishing House of the V.N. Orekhovich Institute of Biomedical Chemistry, 2005. 160 p. (in Russian)

48. Shagiakhmetov F.S., Shamakina I.Y., Davydova T.V. [Mechanisms underlying controlled alcohol consumption: nociceptine/kappa-opioid related gene expression in the rat brain]. Patogenez. [Pathogenesis]. 2018; 16(4): 112-114. DOI: 10.25557/2310-0435.2018.04.112-114 (in Russian)

49. Norman A.L., Pulido C., Squeglia L.M., Spadoni A.D., Paulus M.P., Tapert S.F. Neural activation during inhibition predicts initiation of substance use in adolescence. Drug Alcohol Depend. 2011; 119(3): 216223. DOI: 10.1016/j.drugalcdep.2011.06.019

50. Mahmood O.M., Goldenberg D., Thayer R., Migliorini R., Simmons A.N., Tapert S.F. Adolescents' fMRI activation to a response inhibition task predicts future substance use. Addict. Behav. 2013; 38(1): 1435-1441. DOI: 10.1016/j.addbeh.2012.07.012

51. Rando K., Chaplin T.M., Potenza M.N., Mayes L., Sinha R. Prenatal cocaine exposure and gray matter volume in adolescent boys and girls: relationship to substance use initiation. Biol. Psychiatry. 2013; 74(7): 482-489. DOI: 10.1016/j.biopsych.2013.04.030

52. White C.M. The pharmacologic and clinical effects of illicit synthetic cannabinoids. .J Clin. Pharmacol. 2017; 57(3): 297-304. DOI: $10.1002 /$ jcph.827

53. Lisdahl K.M., Gilbart E.R., Wright N.E., Shollenbarger S. Dare to delay? The impacts of adolescent alcohol and marijuana use onset on cognition, brain structure, and function. Front Psychiatry. 2013; 4: 53. DOI: $10.3389 /$ fpsyt.2013.00053

54. Hammond C. J., Mayes L. C., Potenza M. N. Neurobiology of adolescent substance use and addictive behaviors: prevention and treatment implications. Adolesc. Med. State Art. Rev. 2014; 25(1): 15-32.

55. Montag C., Kirsch P., Sauer C., Markett S., Reuter M. The role of the CHRNA4 gene in Internet addiction: a case-control study. J. Addict. Med. 2012; 6(3): 191-195. DOI: 10.1097/ADM.0b013e31825ba7e7

56. Potenza M.N., Sofuoglu M., Carroll K.M., Rounsaville B.J. Neuroscience of behavioral and pharmacological treatments for addictions. Neuron. 2011; 69(4): 695-712. DOI: 10.1016/j.neuron.2011.02.009

57. Miliano C., Serpelloni G., Rimondo C., Mereu M., Marti M., De Luca M.A. Neuropharmacology of new psychoactive substances (NPS): focus on the rewarding and reinforcing properties of canna- 
bimimetics and amphetamine-like stimulants. Front. Neurosci. 2016; 10: 153. DOI: $10.3389 /$ fnins. 2016.00153

58. Cha H.J., Seong Y.H., Song M.J., Jeong H.S., Shin J., Yun J., Han K., Kim Y.H., Kang H., Kim H.S. Neurotoxicity of synthetic cannabinoids JWH-081 and JWH-210. Biomol. Ther. (Seoul). 2015; 23(6): 597-603. DOI: 10.4062/biomolther.2015.057

59. Karila L., Benyamina A. Synthetic Cannabinoid Use. In: Cannabis Use Disorders. Ed: Montoya I.D., Weiss S.R.B. Springer, Cham; 2019. 163-176 p. DOI: 10.1007/978-3-319-90365-1
60. Akmaev E.G., Aleksandrov A.S., Alchinova I.B., Bocharov E.V., Karganov M.Yu., Kryzhanovskij G.N., Kucheryanu V.G., Magaeva S.V., Morozov S.G., Noskin L.A., Panfilov D.N., Pshennikova M.G., Sarmanaev S.H., Sepiashvili R.I., Syuch N.I., Fisun A.YA., Chuvin B.T. [Sanologiya]. Ed: Kubatiev A.A., Simonenko V.D. M.: Nauka, 2014. 285 p. (in Russian)

61. [Actual problems of Neuroimmunology]. Ed: Kryzhanovskiy G.N., Magaeva S.V., Morozov S.G. M.: Genius media, 2012. 424 p. (in Russian)

\section{Сведения об авторах:}

Анцыборов Андрей Викторович - врач психиатр-нарколог Медицинского центра «Альфа Центр Здоровья» ООО «Медицина АльфаСтрахования»; аспирант кафедры психиатрии Федерального государственного бюджетного образовательного учреждения высшего образования «Ростовский государственный медицинский университет» Министерства здравоохранения Российской Федерации

Камплицкая Оксана Владимировна - кандидат медицинских наук, заведующая 5-м психиатрическим отделением Азовского филиала Государственного бюджетного учреждения Ростовской области «Психоневрологический диспансер»

Овсянников Марк Вадимович - доктор медицинских наук, доцент кафедры психиатрии Федерального государственного бюджетного образовательного учреждения высшего образования «Ростовский государственный медицинский университет» Министерства здравоохранения Российской Федерации

Стадник Кристина Витальевна - аспирант кафедры эпидемиологии Федерального государственного бюджетного образовательного учреждения высшего образования «Ростовский государственный медицинский университет» Министерства здравоохранения Российской Федерации

Панченко Леонид Федорович - доктор медицинских наук, профессор, академик РАН, главный научный сотрудник лаборатории общей патологии нервной системы Федерального государственного бюджетного научного учреждения «Научно-исследовательский институт общей патологии и патофизиологии»; https:// orcid.org/0000-0003-3111-7028 